\title{
不同发育阶段红系造血细胞的生物力学特性 和血液流变特性的变化
}

\section{王金华 孙大公 姚伟娟 曾 柱 文宗曜“*}

(北京大学医学部基础医学院物理教研室, 北京 100083)

\begin{abstract}
摘要用可诱发小鼠贫血的病毒 (anemia-inducing strain friend's virus, FVA)感染 $\mathrm{BALB} / \mathrm{c}$ 小鼠, $15 \mathrm{~d}$ 后取其脾脏, 分离出原红细胞, 在加促红细胞生成素(EPO)等的培养 基中培养 $12,24,48 \mathrm{~h}$, 获得大量相对同步化的早幼红细胞、中幼红细胞、晚幼红细胞, 研究了不同发育阶段的上述早期红系造血细胞的生物力学及血液流变学特性的变化规 律. 发现不同发育阶段的早期红系造血细胞, 随着发育其电泳率、渗透脆性、膜的流 动性和黏弹性都发生了改变, 这种改变与膜脂的组成、膜蛋白、细胞膜的骨架蛋白、脂 质分子与蛋白质分子的相互作用有关.
\end{abstract}

\section{关键词红系造血细胞 电泳率 渗透脆性 膜的流动性 膜的黏弹性}

我们已经研究了红细胞衰老过程中的生物力学特性和血液流变特性 ${ }^{[1,2]}$, 还对由骨髓或脾 脏释放进人外周血循环的最年青红细胞一一网织红细胞的血液流变特性作了研究 ${ }^{[3]}$, 但在国 内外文献中未见对更早时期不同阶段的红系造血细胞(原红细胞、早幼红细胞、中幼红细胞、 晚幼红细胞均是有核细胞)的生物力学特性和血液流变特性的研究报道. 本研究通过对不同发 育阶段的早期红系造血细胞的生物力学特性和血液流变特性进行研究, 以期探索其中的变化 规律.

人们对不同发育阶段的早期红系造血细胞的其他方面作了不少研究，如 Koury 等人 ${ }^{[4]}$ 发 现促红细胞生成素(erythropoietin, EPO)对不同发育阶段的早期红系造血细胞发育有促进作用, 张世馥等人 ${ }^{[5]}$ 发现从不同发育阶段的红系造血细胞提取红细胞分化因子(erythroid differentiation factor, EDF)有诱导细胞分化的作用, Barbe 等人 ${ }^{[6]}$ 发现骨髓内巨噬细胞和早期有核红细胞结合 紧密, 这种结合有利于促进其成熟, 如果通过静脉注射 dichloromethy-enediphosphonale 除去巨 噬细胞, 发现有不成熟的红细胞释放到周边血中, Barnache 等人 ${ }^{[7]}$ 发现 Spi-1(一种转录因子)的 过分表达直接阻止早期有核红细胞的分化. 但是, 在从骨髓或脾脏进人到血液循环系统前的 早期红系造血细胞, 在不同发育阶段其流变特性的变化规律如何呢? 这正是本研究的目的.

\section{1 材料与方法}

\section{1 小鼠脾造血细胞的培养 ${ }^{[4]}$}

将 $0.2 \mathrm{~mL}$ (1: 50 倍稀释)含诱发贫血病毒(FVA)的小鼠血清(FVA 病毒由中国协和医科大

2001-09-06 收稿, 2001-12-17 收修改稿

* 国家自然科学基金(批准号: 39830110)与博士点基金资助项目

** 联系人, E-mail: rheol@ mail.bjmu.edu.cn 
学周建平赠送), 经尾静脉注射到 8 10 周龄的 BALB/c 雌性小鼠. $15 \mathrm{~d}$ 后断颈处死, 无菌条件下 取出脾脏, 在无血清培养基中洗 3 次并剪碎, 用 80 和 200 目钢网过滤, 去除结缔组织后, 将收 集到的脾单细胞悬液平铺在 Ficoll-Urografin 分层液 (1.070) 的液面上, 并使细胞悬液与 Ficoll-Urografin 分层液体积之比为 2: 1 左右, $500 \times g$ 离心 $25 \mathrm{~min}$, 用尖吸管吸取位于两层液 体交界面处的单个核细胞, 在 IMDM 培养液中制成单细胞悬液, 于直径 $15 \mathrm{~cm}$ 塑料培养血中培 养, 细胞密度为 $10^{6}$ 个 $/ \mathrm{mL}$. 培养液 IMDM 含 $30 \%$ 小牛血清, $1 \% \mathrm{BSA}, 100 \mathrm{U} / \mathrm{mL}$ 青電素, 100 $\mu \mathrm{g} / \mathrm{mL}$ 链霉素, $0.2 \mathrm{U} / \mathrm{mL} \mathrm{EPO}, 10^{-4} \mathrm{~mol} / \mathrm{L} \alpha$-硫代甘油, 置于 $37^{\circ} \mathrm{C}$ 和 $5 \% \mathrm{CO}_{2}$ 条件下捊箱培养 12,24 , $48 \mathrm{~h}$ 后, 分别收集细胞. 作细胞涂片, 用 Wright-Giemsa 染液染色, 以鉴定不同发育阶段的细胞, 并用联苯胺染色以分析其分化产物血红蛋白的染色反应.

\section{2 细胞电泳率的测定}

用 $9 \%(w / v)$ 蔗糖溶液将细胞配成 $2 \times 10^{6}$ 个 $/ \mathrm{mL}$ 的悬液, 在细胞电泳仪(LIANG- 100 , 上海医 科大学生产)测定其电泳率(电压 $40 \mathrm{~V}, 30^{\circ} \mathrm{C}$ ), 选取每 10 个细胞一组进行测量, 求平均值.

\section{3 细胞渗透脆性的测定}

用 $\mathrm{PBS}(\mathrm{pH} 7.4,300 \mathrm{mOsm} / \mathrm{kg}$ ) 和蒸馏水以不同比例混合, 配成 11 种不同渗透压的 PBS 溶 液, 渗透压范围在 0 300 mOsm $/ \mathrm{kg}$ 之间. 将细胞配成 $2 \times 10^{6}$ 个 $/ \mathrm{mL}$ 的悬液, 将悬液以每份 $100 \mu \mathrm{L}$ 分别置于 $\mathrm{Ep}$ 管中, 离心 $(300 \times g, 5 \mathrm{~min})$ 后弃上清, 再分别加人 $500 \mu \mathrm{L}$ 不同渗透压的 $\mathrm{PBS}$ 溶液, 充分混匀. 用细胞计数板计各管中的完整细胞数, 以 $300 \mathrm{mOsm} / \mathrm{kg}$ 管的细胞数为标准, 其他管 中的细胞数除以标准细胞数得到各种渗透压下的未破碎细胞百分数, 绘制渗透脆性曲线.

\section{4 细胞黍弹性的测定}

微吸管系统由倒置显微镜 (重庆光学 仪器厂生产)、显微操作仪、图像处理器、 压力控制记录系统和微吸管等部分组成 (图 1). 将 $0.5 \mathrm{~mL}$ 细胞悬液 $\left(1 \times 10^{6}\right.$ 个 $\left./ \mathrm{mL}\right)$ 注人特定小室内, 置于倒置显微镜载物 台上. 采用内直径为 $2.41 \sim 3.13 \mu \mathrm{m}$ 的微 吸管, 利用显微操作仪控制微吸管尖部 靠近细胞表面, 打开压力控制与记录系 统并通过微吸管给细胞施以一定的阶跃 负压, 使细胞的一小部分吸人微管内. 利用图像记录仪每隔 $120 \mathrm{~ms}$ 记录一帧图

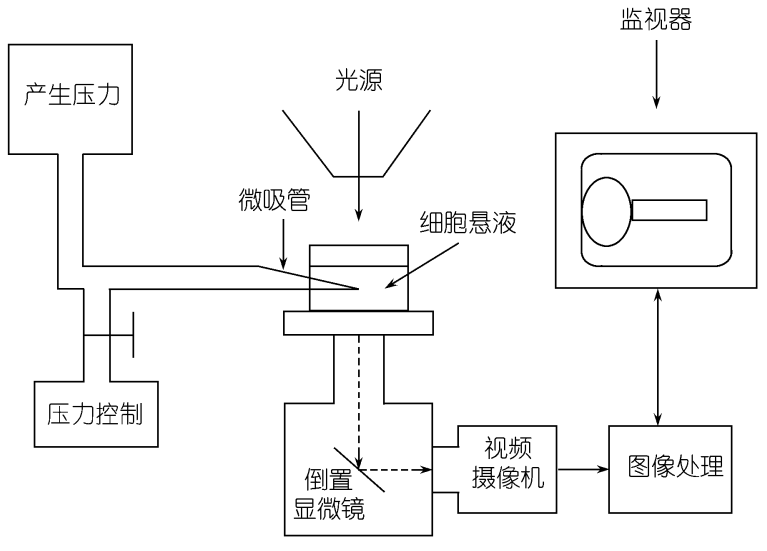

图 1 微吸管系统的方框图

像, 从而可测量到不同时间内细胞被吸人管内的变形值, 实验在室温 $\left(30^{\circ} \mathrm{C}\right)$ 下进行 ${ }^{[8,9]}$. 分析结 果采用标准固体线性黏弹性模型(图 2), 该模型由 Sung 等人 ${ }^{[10]}$ 根据白细胞黏弹性的实验结果 和理论分析提出, 由二条平衡的臂组成, 其中一臂为弹性系数为 $K_{1}$ 的弹性元件, 另一臂则由 弹性系数 $K_{2}$ 的弹性元件与黏性系数为 $\mu$ 的黏性元件串联组成, 其本构方程如下:

$$
\sigma+\left(\mu / K_{2}\right) \partial \sigma / \partial t=K_{1} \varepsilon+\mu\left(1+K_{1} / K_{2}\right) \partial \varepsilon / \partial t,
$$

其中 $\sigma$ 和 $\varepsilon$ 分别为应力和应变, $\partial \sigma / \partial t, \partial \varepsilon / \partial t$ 分别为应力和应变时间的偏导数, 弹性系数 $K_{1}$, $K_{2}$ 与时间无关, 主要反映了细胞的初始变形程度, 黏性系数 $\mu$ 反映了细胞变形对时间的依赖 性. 在本研究设计的的蠕变力学实验中, 细胞蠕变特性由时间常数 $\mu\left(\left(1 / K_{1}\right)+\left(1 / K_{2}\right)\right)$ 确定, 最 


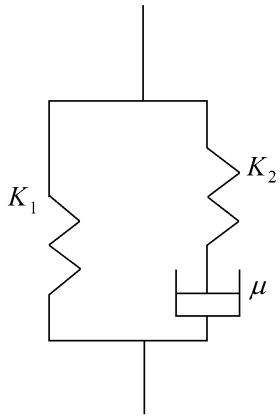

图 2 标准固体线性黏弹模型示意图 $K_{1}, K_{2}$ 为弹性系数, $\mu$ 为黏性系数

大变形值正比于 $1 / K_{1}$.

\section{5 荧光偏振测膜的流动性}

取不同发育阶段的早期红系造血细胞分别置于 PBS 中, 配成 $2 \times 10^{6}$ 个 $/ \mathrm{mL}$. 然后加等体积的 $2 \times 10^{-6}$ $\mathrm{mol} / \mathrm{L} \mathrm{DPH}$ 一起在室温孵育 $30 \mathrm{~min}$, 然后以 $1000 \mathrm{r} / \mathrm{min}$ 离心 $10 \mathrm{~min}$, 弃去上清, 再用等渗 PBS 液体洗涤 3 次, 加等渗 PBS 缓冲液至终体积为 $2 \mathrm{~mL}, 25^{\circ} \mathrm{C}$ 用苂光分光 光度计(HITACHI 850 型)测苂光偏振度 $P^{[11]}$ :

$$
P=\frac{I_{\mathrm{VV}}-G I_{\mathrm{VH}}}{I_{\mathrm{VV}}+G I_{\mathrm{VH}}},
$$

其中 $I_{\mathrm{VV}}$ 为起偏器和检偏器光轴均在垂直方向时的苂 光强度, $I_{\mathrm{VH}}$ 为起偏器光轴垂直方向、检偏器光轴在水平方向时的苂光强度; $G$ 为校正因子, 校 正由于单色器所产生的附加偏振, 按 Azumi 法: $G=I_{\mathrm{HV}} / I_{\mathrm{HH}}$, 其中 $I_{\mathrm{HH}}$ 为起偏器、检偏器光 轴均在水平方向时的苂光强度, $I_{\mathrm{HV}}$ 为起偏器光轴在水平方向、检偏器光轴在垂直方向的菼光 强度, 求得 $P$ 值后, 进一步算出表征细胞膜流动性的微黏度 $\eta=\frac{2 P}{0.46-P}$. 微黏度越大, 流动性 越小; 微黏度越小, 流动越大. 激发波的波长为 $362 \mathrm{~nm}$, 发射波的波长为 $432 \mathrm{~nm}$, 标记的细胞 在 $2 \mathrm{~h}$ 之内测完, 整个过程要求细胞存活.

\section{2 结果}

\section{1 细胞的 Wright-Giemsa 染色图和联苯胺染色图}

由图 3 可以看出, 从原红细胞到晚幼红细胞, 核由大到小, 核仁从有到无, 细胞质由少到 多, 在晚幼红阶段, 细胞核处于偏核和排核的情况.

由图 4 可以看出, 原红细胞和早幼红细胞的联苯胺的染色基本上为阴性, 中幼红细胞的联 苯胺染色体为弱阳性, 晚幼红阶段为阳性.

\section{2 细胞电泳率}

小鼠成熟红细胞、原红细胞、早幼红细胞、中幼红细胞和晚幼红细胞的电泳率如表 1 所 示, 小鼠原红细胞、早幼红细胞和中幼红细胞电泳率和小鼠正常红细胞的差别比较大, 小鼠的 晚幼红细胞也有差别, 但不及它们明显, 说明早期不同阶段红系造血细胞在分化过程中其表 面电荷逐渐减小.

\section{3 细胞渗透脆性}

小鼠原红细胞、早幼红细胞、中幼红细胞、晚幼红细胞、成熟红细胞的渗透脆性曲线如 图 5 所示. 除成熟红细胞外, 4 种细胞渗透性曲线差别不是很明显, 它们与红细胞的渗透脆性 曲线主要区别表现在: ( i ) $220 \mathrm{mOsm} / \mathrm{kg}$ 左右时, 成熟红细胞的破裂百分数在 $4.5 \%$, 而其他 4 种细胞达 11\% 19\%, 成熟红细胞抵抗渗透能力最强, 其中原红细胞最差, 晚幼红细胞稍好. (ii ) 在非常低的渗透压情况下，其他 4 种有核细胞不破裂的百分数多于成熟红细胞.

\section{4 膜的流动性}

小鼠原红细胞、早幼红细胞、中幼红细胞、晚幼红细胞、成熟红细胞的膜苂光偏振度 $P$ 值 


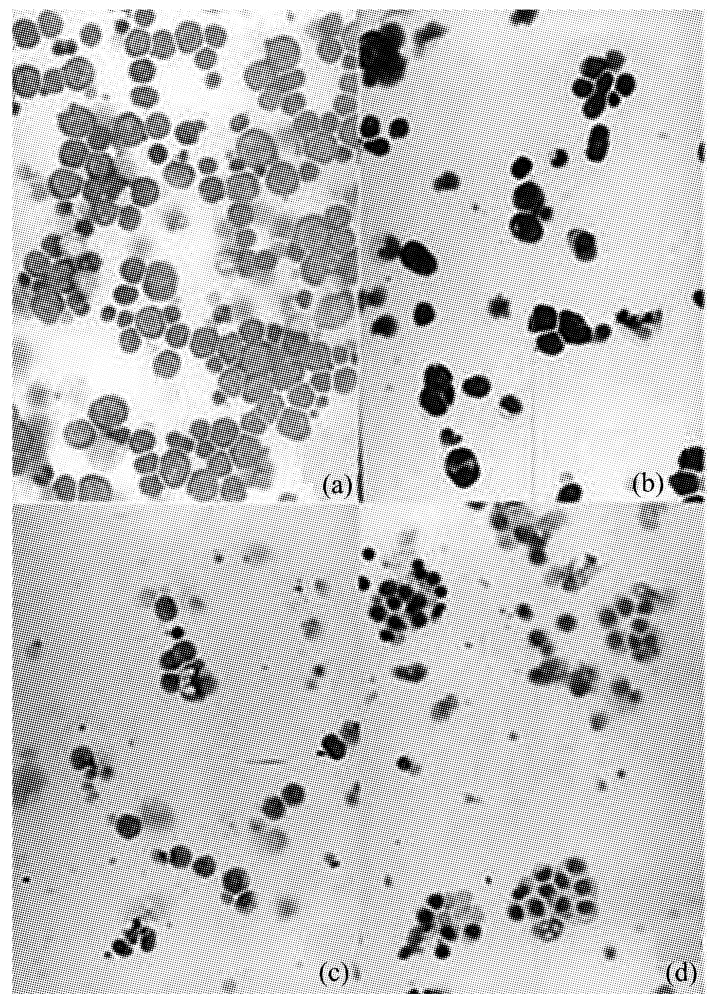

图 3 早期有核红细胞的 Wright-Giemsa 染色图 (a) 原红细胞; (b) 早幼红细胞; (c) 中幼红细胞; (d) 晚幼红细胞

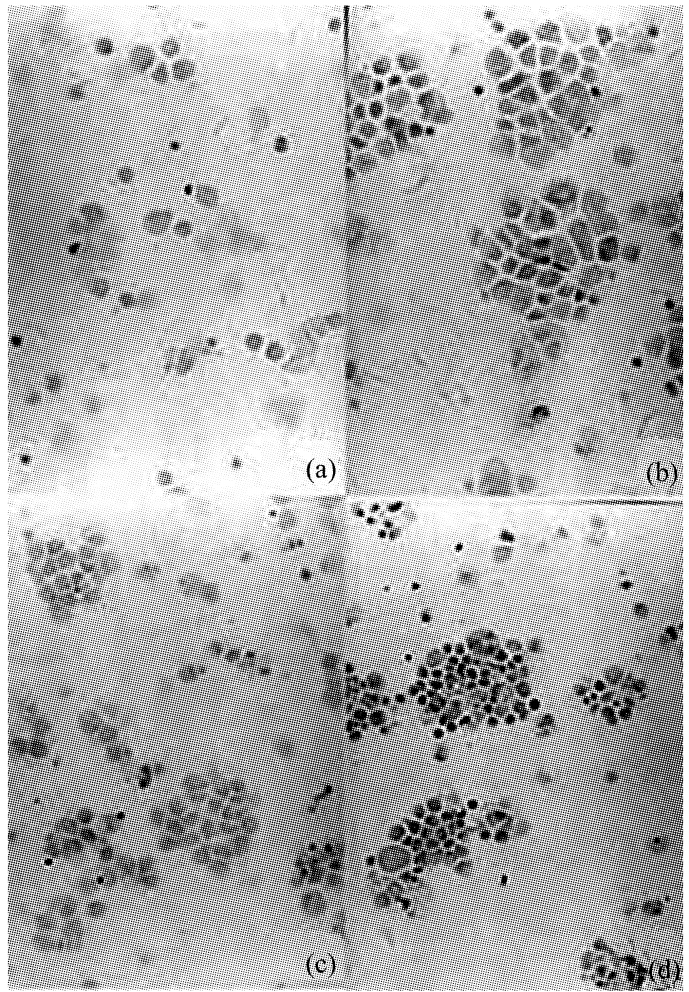

图 4 早期有核红细胞的联苯胺染色图

（a）原红细胞; (b) 早幼红细胞; (c) 中幼红细胞; (d) 晚幼 红细胞

表 1 不同发育阶段早期红系造血细胞和成熟红细胞的电泳率 ${ }^{\text {a) }}(\bar{X} \pm \mathrm{SD})$

\begin{tabular}{cccccc}
\hline 200 & 原红细胞 & 早幼红细胞 & 中幼红细胞 & 晚幼红细胞 & 成熟红细胞 \\
\hline 电泳率 $/ \mu \mathrm{m} \cdot \mathrm{s}^{-1} \cdot \mathrm{V}^{-1} \cdot \mathrm{cm}^{-1}$ & $1.618 \pm 0.024^{\mathrm{a})}$ & $1.534 \pm 0.026^{\mathrm{a})}$ & $1.436+0.031^{\mathrm{a})}$ & $1.293 \pm 0.044^{\mathrm{a})}$ & $1.233 \pm 0.032$ \\
\hline
\end{tabular}

a) 同成熟红细胞相比, $P<0.01$

和由此算出的微黏度值如表 2 所示.

\section{5 细胞的黍弹性}

单个细胞在 $196 \mathrm{~N} / \mathrm{m}^{2}$ (2 $\mathrm{cm}$ 水柱)的阶跃负 压作用下，开始有一个快速响应，然后随着时间 的增加, 其在微吸管内的部分缓慢伸长, 如图 6 如示, 根据标准固体线性黏弹性模型, 最初快速 响应的变形值与细胞的弹性系数 $K_{1}$ 有关, 而随后 的蠕变过程则可由弹性系数 $K_{2}$ 和黏性系数 $\mu$ 进行 描述, 将测量实验结果用方程(1)进行非线性迭代 方法拟合, 得到 4 种细胞的黏弹性系数(表 3 ), 其中 红细胞的弹性系数和黏性系数引自文献 $[12$, 13](红细胞无细胞核, 不适合这种模型).

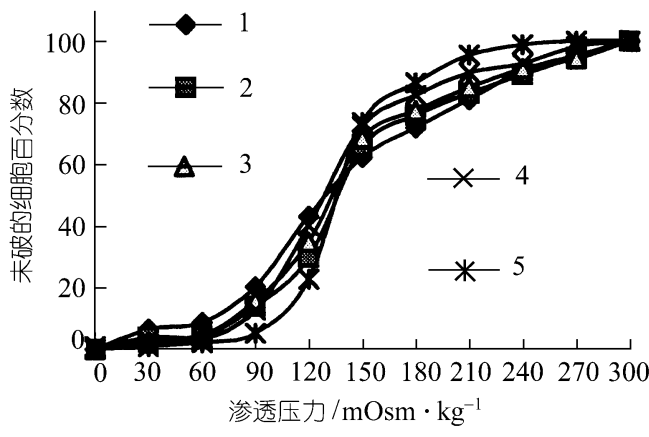

图 5 不同发育阶段早期红系造血细胞和成熟 红细胞的渗透脆性曲线

1 示原红细胞; 2 示早幼红细胞; 3 示中幼红细胞; 4 示晚幼 红细胞; 5 示成熟红细胞 
表 2 不同发育阶段的早期红系造血细胞和成熟红细胞膜的苂光偏振度与微黏度 $\left(25^{\circ} \mathrm{C}\right)(\bar{X} \pm \mathrm{SD})$

\begin{tabular}{ccc}
\hline & 苂光偏振度 $\mathrm{P}$ & 微黏度 $\eta(\mathrm{Pa} \cdot \mathrm{s})$ \\
\hline 原红细胞 & $0.256 \pm 0.001$ & $2.511 \pm 0.03^{\mathrm{a})}$ \\
早幼红细胞 & $0.252 \pm 0.001$ & $2.421 \pm 0.03^{\mathrm{a})}$ \\
中幼红细胞 & $0.250 \pm 0.001$ & $2.38 \pm 0.03^{\mathrm{a})}$ \\
晚幼红细胞 & $0.248 \pm 0.003$ & $2.34 \pm 0.02^{\mathrm{a}}$ \\
成熟红细胞 & $0.243 \pm 0.002$ & $2.240 \pm 0.04$ \\
\hline
\end{tabular}

a) 同成熟红细胞相比, $P<0.01$

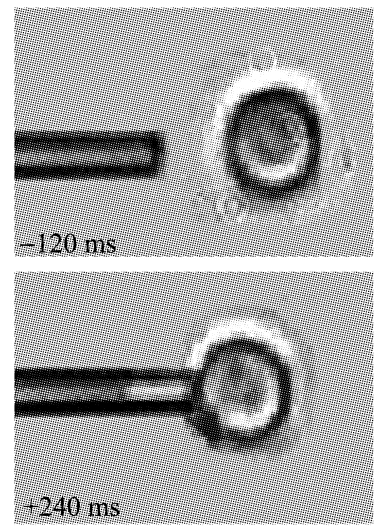

图 6 早期有核红细胞在施加压力的作用下，细胞变形被吸人管的渐进系列图 微吸管压力为 $196 \mathrm{~N} / \mathrm{m}^{2}$

表 3 不同发育阶段的早期红系造血细胞和成熟红细胞的弹性系数和黏度系数 $(\bar{X} \pm S D)$

\begin{tabular}{|c|c|c|c|c|c|}
\hline & 原红细胞 & 早幼红细胞 & 中幼红细胞 & 晚幼红细胞 & 成熟红细胞 \\
\hline$K_{1} / \mathrm{N} \cdot \mathrm{m}^{-2}$ & $19.71 \pm 2.8^{\mathrm{a})}$ & $12.25 \pm 1.57^{\mathrm{a})}$ & $6.93 \pm 1.02^{\mathrm{a})}$ & $(5.6 \pm 0.75) \times 10^{-2 \mathrm{a})}$ & $4.2 \times 10^{-4}$ \\
\hline$K_{2} / \mathrm{N} \cdot \mathrm{m}^{-2}$ & $6.83 \pm 0.92$ & $5.62 \pm 0.62$ & $4.52 \pm 0.75$ & $(2.2 \pm 0.32) \times 10^{-2}$ & \\
\hline$\mu / \mathrm{N} \cdot \mathrm{S} \cdot \mathrm{m}^{-2}$ & $8.9 \pm 1.2$ & $7.4 \pm 0.93$ & $5.4 \pm 1.1$ & $(1.8 \pm 0.26) \times 10^{-2}$ & $4 \times 10^{-5}$ \\
\hline
\end{tabular}

a) 同成熟红细胞相比, $P<0.01$

\section{3 讨论}

通过对不同发育阶段的早期红系造血细胞的生物流变特性的测定，可以探索早期有核红 细胞在不同发育阶段的微流变学特性变化规律，如细胞电泳率、渗透脆性、膜的黏弹性、膜的 流动性如何变化等等.

细胞电泳实验表明，随着不同发育阶段的早期红系造血细胞不断成熟，其电泳率不断减 少, 说明其表面电荷不断减少, 即表面唾液酸减少, 这与 Dason 等人用电子显微镜观察处于不 同生长期的红细胞的结果类似, 即老年与年幼红细胞膜有着明显的差异, 老化的红细胞因膜 糖链上的唾液酸结构有所改变, 表现在其表面负电荷较年幼红细胞减少. 我们对于不同发育 阶段的早期红系造血细胞的电泳率的测量发现了类似的变化规律. 由此可见，在红系生成到 出骨髓或脾脏后变成红细胞的整个生命周期中，其表面电荷总是单调的减少.

从渗透脆性实验看出, 成熟红细胞在 260 300 mOsm/ $\mathrm{kg}$ 正常渗透压范围内能保持较完整 的形态，破裂数目很少，而与其他不同发育阶段红细胞比较稍差. 当渗透压减少在 220 $\mathrm{mOsm} / \mathrm{kg}$ 以下时, 红细胞破裂数远小于其他不同发育阶段的早期, 说明成熟红细胞比不同发 
育阶段的早期红细胞有较强的抵抗能力, 而在非常低的渗透压力下, 不同发育阶段的早期红 细胞的未破裂数都高于成熟红细胞, 这可能与它们含有细胞核, 在非常低的渗透压力下有较 强的抗渗透脆性能力有关.

由膜的流动性实验得知，微黏性随着不同发育阶段红系造血细胞向下分化而减小，说明 膜的流动性不断升高, 但与成熟红细胞的流动性相比仍然存在明显的差异, 只有当发育成熟 后其流变指标逐渐完善, 以确保其应有的正常生理功能.

微吸管吸㓍技术是公认的测量单个细胞的最重要的技术之一, 此种方法比较精确. 我们 用微吸管吸吮技术分别测量了原红细胞、早幼红细胞、中幼红细胞和晚幼红细胞, 发现 $K_{1}$ 明 显减少, $K_{2}$ 与 $\mu$ 无明显变化. 由于细胞的最大变形值正比于 $1 / K_{1} . K_{1}$ 的减少说明细胞的最大变 形能力变好, 这与其内部的核不断缩小有密切关系, 细胞核小, 变形能力强, 细胞核大, 变形 能力差. 上述原红细胞、早幼红细胞、中幼红细胞和晚幼红细胞变形性逐渐增强的这一变化规 律与它们发育成熟后通过骨髓或脾脏进人血液循环系统, 直到微循环系统完成气体交换与养 料输运, 通过脾窦的选择的完美的生理功能相符合. 在病理生理情况下的溶血性贫血病、白血 病等, 一些未成熟早期的有核红细胞进入血液循环, 这些细胞在血流中破坏几率大增, 寿命短, 又从相反的方面证明了上述结论. 此外, 晚幼红细胞相比前 3 种细胞, 变形值相差大而且相对 更接近于成熟红细胞, 这可能与其偏核和逐渐排核增强其变形能力有关. 在病理情况下, 它是 周围血涂片中最常见的有核红细胞, 这可能与晚幼红细胞变形能力增强, 容易穿过内皮细胞 和血窦有关.

总之, 未进人血液循环前的红系造血细胞在骨髓或脾脏中发育的短短约 $48 \mathrm{~h}$ 内, 这些含 有细胞核的幼稚红细胞经历了原红细胞、早幼红细胞、中幼红细胞及晚幼红细胞等不同的阶 段, 在不同的阶段, 其微流变特性及生物力学特性都有差异. 在红系细胞生命周期中, 这段时 间新陈代谢较为活跃, 变化较为明显. 这类有核红细胞将再经过约 $48 \mathrm{~h}$, 完成从网织红细胞到 新生红细胞的另一活跃的新陈代谢的飞跃阶段, 然后进人一个比较稳定的(约 $35 \mathrm{~d})^{[14]}$ 生命周期, 直到生命后半期的较快的衰老阶段. 不同发育阶段的早期红系造血细胞随着发育其电泳率、渗 透脆性、膜的流动性和黏弹性都发生了改变, 这种改变与膜脂的组成、膜蛋白、细胞膜的骨架 蛋白、脂质分子与蛋白质分子的相互作用有关，提示随着细胞发育的不断成熟，其生化、生物 物理特性都会发生了明显变化. 显然, 对于早期红系造血细胞发育阶段微观流变特性的研究, 除了对于认识细胞的本身的生长发育具有重要意义外, 还增强了我们对溶血性贫血病、再生障 碍性贫血、遗传性球形细胞增多症、白血病等各种血液病的机理的认识, 有利于提出正确的治 疗方案.

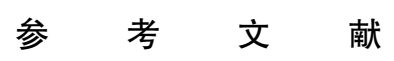

1 Wen Z Y, Song L C, Yan Z Y, et al. An animal model to study erythrocyte senescence with a narrow time window: alterations in osmotic fragility and deformability of erythrocytes during their life span. Clinical Hemorhology and Microcirculation, 1998, 18(1): 299 306

2 Wen Z Y, Song L C, Yan Z Y, et al. An animal model to study erythrocyte senescence with a narrow time window of erythrocyte production. Clinical Hemorhology and Microcirculation, 1998, 18(1): 75 84

3 谢利德, 孙大公, 姚伟娟, 等. 在体网织红细胞微观流变学特性的研究. 中国科学, C辑, 2001, 31(1): 78 83 
4 Koury M J, Sawyer S T, Bondurant M C. Splenic erythroblast in anemia-inducing friend disease: a source of cells for studies of erythropoietin-mediated of differentiation. J Cell Physiol, 1984, 121(3): 526 532

5 张世馥, 王 金金, 刘 䑣, 等. 不同发生阶段红系血细胞中红细胞分化例子的检测. 解剖学报, 1994, 25: 156 160

6 Barbe E, Huitinga I, Dopp E A, et al. A novel bone marrow frozen section assay for studying hematopoietic interation in situ: the role stromal bone marrow macrophages in erythroblast binding. J Cell Sci, 1996, 109(pt,12): 2937 2945

7 Barnache S, Wendling F, Lacombe C, et al. Spi-1 transgenic mice develop a clonal erythroleukemia which does not depend on p53 mutation. Oncogene, 1998, 16(23): 2989 2995

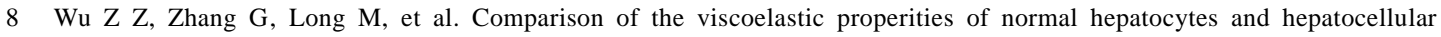
carcinoma cells under cytoskeletal perturbation. Biorheology, 2000, 37: 279 290

9 龙 勉, 吴泽志, 王红兵, 等. 肝细胞粘弹性实验研究. 生物物理学报, 1996, 12: 169 172

10 Schmid G W, Sung K L. Passive mechanical properties of human leukocytes. Biophys J, 1981, 36: 243 256

11 林克椿, 吴本阶. 医学生物物理学. 北京: 北京医科大学, 中国协和医科大学联合出版社. 1999. 74 76

12 Hochmuth R M, Worthy P R, Evans E A. Red cell extensional recovery and the determination of membrane viscosity. Biophys J, 1979, 26: 101 114

13 Chien S, Sung K L, Skalak R, et al. Theoretical and experimental studies on viscoelastic properties of erythrocyte membrane. Biophys J, 1978, 24: 463 487

14 Wandersee N J, Lee J C, Deveau S A, et al. Reduced incidence of thrombosis in mice with hereditary spherocytosis following neonatal treatment with normal hepatopoietic cell. Blood, 2001, 97: 3972 3975 